\title{
Infantile hemangiomas are arrested in an early developmental vascular differentiation state
}

\author{
Soheil S Dadras ${ }^{1,2}$, Paula E North ${ }^{3}$, Jennifer Bertoncini ${ }^{1}$, Martin C Mihm² and \\ Michael Detmar ${ }^{1}$ \\ ${ }^{1}$ Cutaneous Biology Research Center and Department of Dermatology, Massachusetts General Hospital and \\ Harvard Medical School, Charlestown, MA, USA; ${ }^{2}$ Department of Pathology, Massachusetts General Hospital \\ and Harvard Medical School, Boston, MA, USA and ${ }^{3}$ Department of Pathology, University of Arkansas for \\ Medical Sciences and Arkansas Children's Hospital, Little Rock, AR, USA
}

\begin{abstract}
Infantile hemangiomas, the most common tumors of infancy, are vascular tumors characterized by rapid proliferation of endothelial cells during the first few months of postnatal life followed by slow spontaneous involution, whose molecular pathogenesis remains unclear. The recent identification of developmental expression of vascular lineage-specific markers prompted us to characterize infantile hemangiomas for the expression of lymphatic endothelial hyaluronan receptor-1 (LYVE-1), Prox-1, CD31 and CD34. We found that LYVE-1, a specific marker for normal and tumor-associated lymphatic vessels, was strongly expressed in tumor cells of infantile hemangiomas $(n=28)$, but not in other vascular tumors including pyogenic granulomas $(n=19, P<0.0001)$ or intramuscular hemangiomas $(n=9)$, using LYVE-1/CD31 double immunostains. Whereas LYVE-1 expression was detected on the endothelial cells of all proliferating infantile hemangiomas, this lymphatic marker was absent from the lesional capillaries during involution in the majority of cases $(P=0.0009)$. The majority of LYVE-1 ${ }^{+}$endothelial cells also expressed CD34, but were negative for the lymphatic-specific homeobox protein Prox-1. Based on coexpression of both LYVE-1 and the blood vascular marker CD34, we propose that the endothelial cells in proliferating infantile hemangioma are arrested in an early developmental stage of vascular differentiation. The immature, incompletely differentiated immunophenotype of proliferating infantile hemangiomas may contribute to their rapid growth during the first few months of life.
\end{abstract}

Modern Pathology (2004) 17, 1068-1079, advance online publication, 14 May 2004; doi:10.1038/modpathol.3800153

Keywords: infantile hemangioma; LYVE-1; Prox1; lymphangiogenesis

Infantile hemangiomas, the most common tumor of infancy, are benign vascular proliferations composed of densely packed capillaries composed of endothelial cells and pericytes expanding in a lobular pattern. ${ }^{1}$ In contrast to vascular malformations, infantile hemangiomas are usually absent or inconspicuous at birth and are characterized by a remarkably predictable biologic behavior: rapid postnatal proliferation and slow spontaneous involution. ${ }^{2}$ Involution is characterized by apoptosis and disappearance of capillaries, with replacement by loose fibrous or fibro-fatty tissue. Infantile hemangiomas occur more commonly in premature Caucasian female infants, ${ }^{3}$ but are distinguished

Correspondence: Dr SS Dadras, MD, PhD, Cutaneous Biology Research Center and Department of Pathology, Massachusetts General Hospital, Building 149, 13th Street, Charlestown, MA 02129, USA.

E-mail: sam.dadras@cbrc2.mgh.harvard.edu

Received 5 February 2004; revised 19 March 2004; accepted 23 March 2004; published online 14 May 2004 from other vascular tumors and malformations by their specific expression of erythrocyte-type glucose transporter protein, GLUT-1. ${ }^{4}$ The molecular pathogenesis of infantile hemangiomas remains a mystery to date, partly due to a confusing nomenclature of vascular birthmarks ${ }^{5}$ and to the lack of lineagespecific vascular differentiation markers.

Several recent studies have investigated the cellular and molecular basis of these tumors, leading to the proposal of distinct but controversial mechanisms of pathogenesis. It has been proposed that infantile hemangiomas are due to the clonal proliferation of endothelial cells based on the analysis of X-chromosome inactivation patterns, indicating that a single progenitor cell may give rise to the infantile hemangioma endothelial cell population. ${ }^{6,7}$ Most recently, it was found that this population of endothelial progenitor cells coexpresses CD34 and the progenitor cell marker CD133, as detected by flow cytometry in 11/12 proliferating infantile hemangioma specimens. ${ }^{8}$ It has also been suggested that infantile hemangiomas 

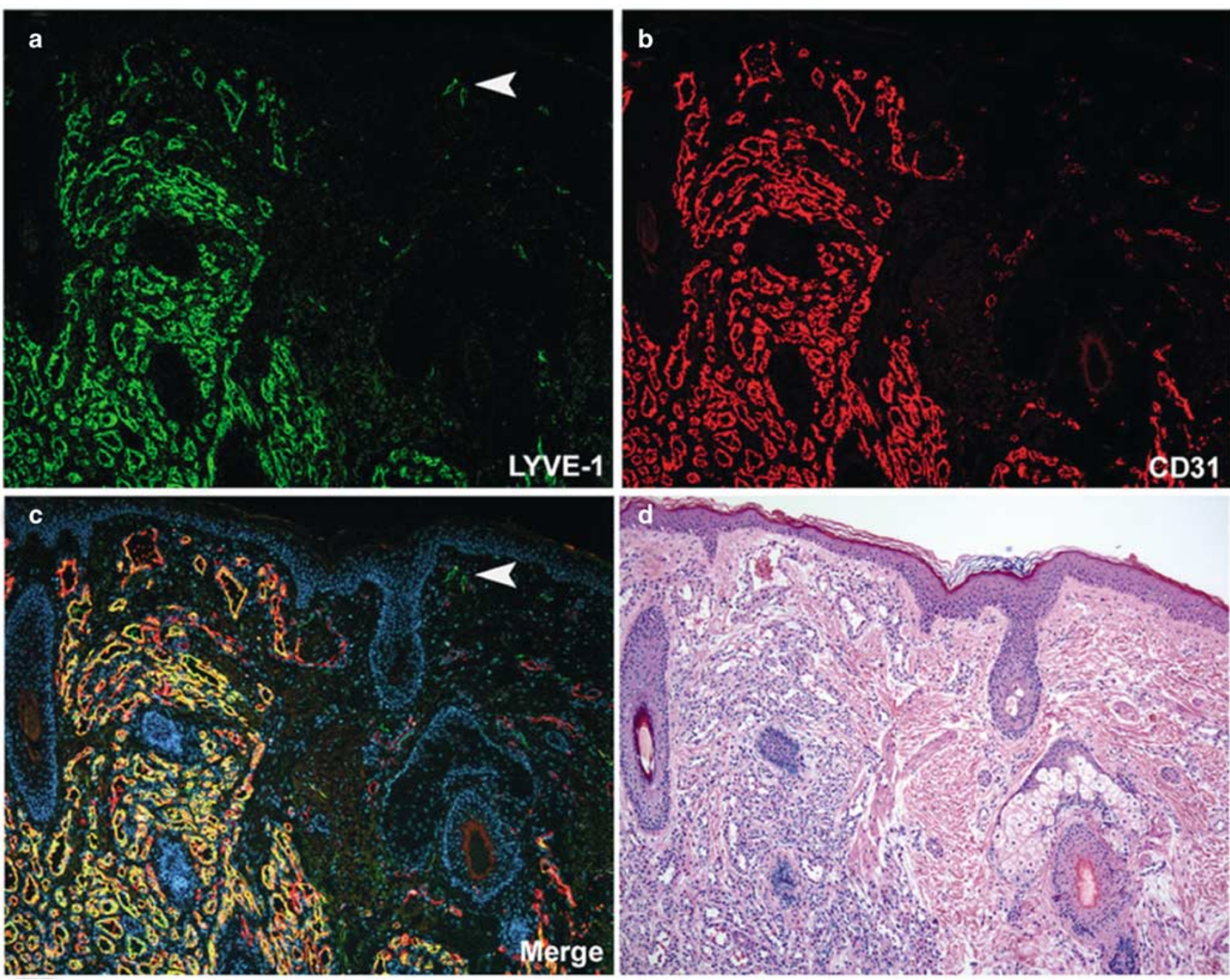

Figure 1 LYVE-1 is expressed by endothelial cells of proliferating infantile hemangiomas. (a) and (b) Immunofluorescent stains for LYVE1 (green) and CD31 (red) demonstrate overlapping reactivity in a lesion from the scalp of an 8-month-old female infant. (c) Merged images for LYVE-1 and CD31 show complete overlap (yellow) except for normal lymphatic vessels (arrowhead). (d) Histology of a serial section reveals marked proliferation of capillaries in a lobular configuration (left). (e) Double stains for LYVE-1 and CD31 of a lesion from the lower lip of a 5-month-old female infant show a lesser degree of overlap in lesional capillaries at the infiltrating tumor edge (arrows); native lymphatic vessels (arrowhead) are seen away from the tumor. (f) Histology of a serial section shows tumor lobule near salivary glands (right). (g) Double stains for LYVE-1 and CD31 of a lesion from the ear of a 3-month-old female infant, a tumor frozen section shows extensive overlap. Intraseptal blood vessels (arrows) are CD31 positive, but LYVE-1 negative. (h) A high magnification details the cytoplasmic and membrane distribution of LYVE-1 in lesional capillaries (asterisks); some tumor vessels are negative for LYVE-1 (arrowheads). Cell nuclei are counterstained (blue) with Hoechst (c, e and h). Original magnifications: $\times 100(\mathbf{a})-(\mathbf{d}) ; \times 200(\mathbf{e})-(\mathbf{f})$; and $\times 400(\mathbf{h})$

are the products of somatic mutations in one or more genes that regulate endothelial cell growth. ${ }^{9}$ Although the vast majority of infantile hemangiomas occur sporadically, several kindreds have been identified to segregate as an autosomal dominant trait with high penetrance, and FGFR4, PDGFRB and Flt-4 have been suggested as candidate genes for familial infantile hemangioma. ${ }^{10}$ Somatic mutations of the kinase domain of the VEGFR2 (FLK1/KDR) gene and of the VEGFR3 (FLT4) gene were found in proliferating infantile hemangiomas. ${ }^{7}$ These genetic mutations may result in abnormal endothelial cell proliferation and dysregulation of angiogenesis, as evidenced by the activation of the angiopoietin/Tie2 pathway in isolated endothelial cells from prolifer- ating infantile hemangiomas. ${ }^{11}$ Stimulators of angiogenesis such as VEGF, ${ }^{12}$ bFGF, ${ }^{13}$ monocyte chemoattractant protein-1 ${ }^{14}$ and insulin-like growth factor 2 (IGF-2) ${ }^{15}$ are also upregulated in proliferating infantile hemangioma.

In contrast, tissue inhibitor of metalloproteinase1 , an angiogenesis inhibitor, ${ }^{12}$ and interferon-induced genes were found to be increased during the involution phase. ${ }^{15}$ The induction of interferon genes provides a clinical rationale for the treatment of life-threatening infantile hemangiomas with recombinant interferon- $\alpha-2 a$, an inhibitor of angiogenesis $^{16}$ and may explain recently reported observations of rapid involution of infantile hemangiomas after topical applications of the 

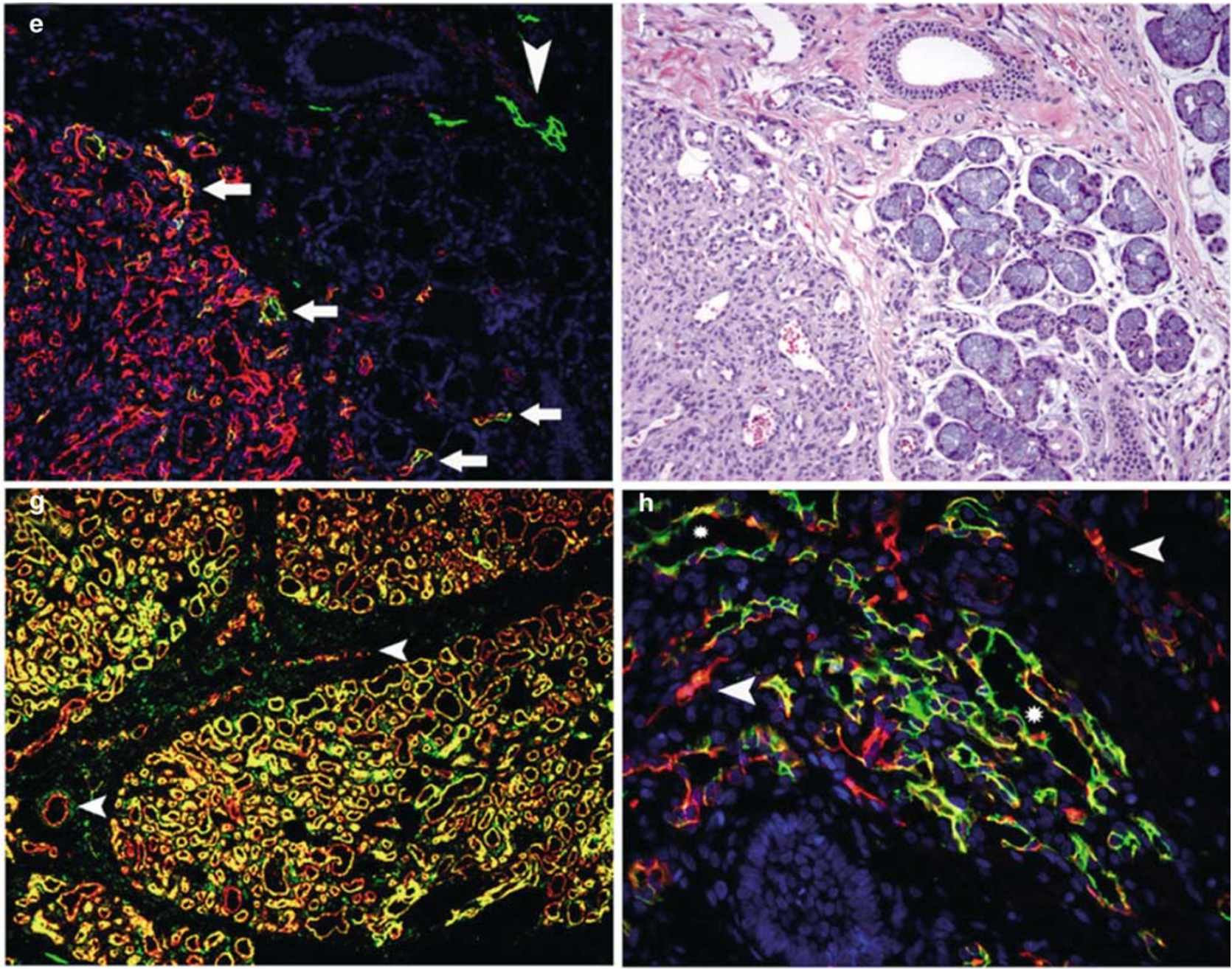

Figure 1 Continued.

immune modulator imiquimod. ${ }^{17}$ Interestingly, the epidermis overlying proliferating infantile hemangioma demonstrates reduced interferon- $\beta$ immunostaining, compared with the epidermis of normal skin, implicating possible paracrine effects of nonendothelial cells in the pathogenesis of infantile hemangioma. ${ }^{18}$ Recent studies have demonstrated that the vessels of infantile hemangioma and placenta share a unique immunophenotype, as evidenced by expression of GLUT-1, merosin, Fc $\gamma$ RII and Lewis Y antigen, leading to a proposal that the precursor cells of infantile hemangioma may originate from placentally derived cells or angioblasts switched to the placental-type vascular phenotype. ${ }^{19}$

In the present study, we investigated the vascular lineage-specific differentiation of proliferating and of involuting infantile hemangiomas, using several of the newly identified blood vascular and lymphatic endothelial cell markers. We analyzed 62 vascular lesions for the expression of the panendothelial marker CD31, and of the lymphatic endothelial hyaluronan receptor-1 (LYVE-1), a $60 \mathrm{kDa}$ integral membrane glycoprotein, considered to be a specific marker for normal ${ }^{20}$ and tumorassociated lymphatic vessels. ${ }^{21,22}$

\section{Materials and methods}

\section{Patient Population and Histological Analyses}

All vascular lesions were excised at Arkansas Children's Hospital, Little Rock, during the past 5 years (1999-2003), after informed consent and institutional review board approval were obtained. These vascular lesions included infantile hemangiomas $(n=28)$, pyogenic granulomas (PG) $(n=19)$, intramuscular hemangiomas (small vessel type, $n=9$ ), umbilical hemangiomas $(n=2)$, congenital nonprogressive hemangioma $(n=1)$, synovial vascular malformation $(n=1)$ and cherry hemangioma $(n=1)$ (Table 1). Patients (age range 2 months to 5 years) with both proliferating $(n=19$, mean age 7.8 months) and involuting $(n=9$, mean age 26.3 months) infantile hemangioma were well represented, with sex and site distribution similar to those reported in large epidemiological studies. ${ }^{23}$ 

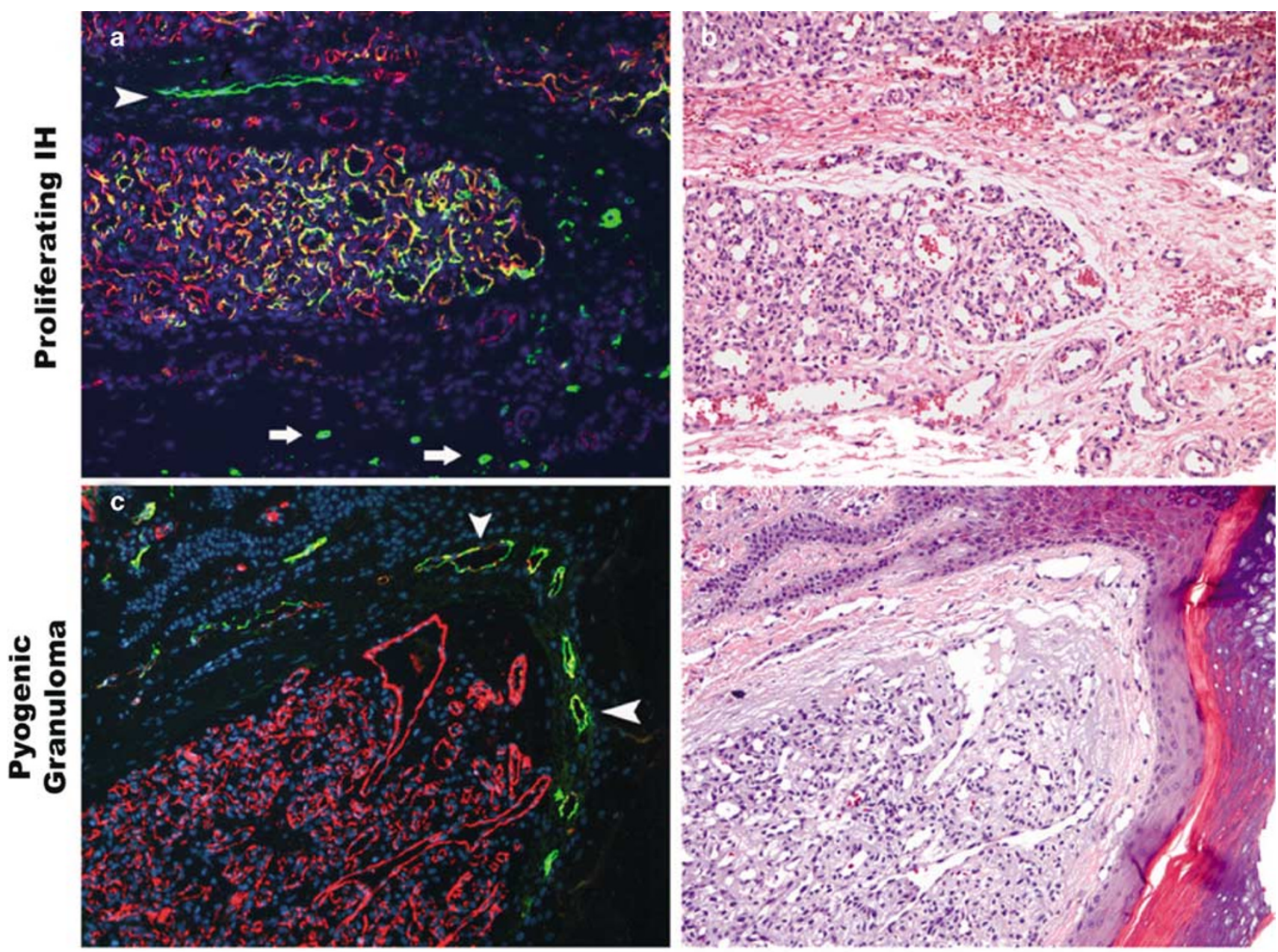

Figure 2 LYVE-1 expression is specific to the endothelial cells of proliferating infantile hemangiomas. (a) Double immunofluorescent stains for LYVE-1 (green) and CD31 (red) show extensive overlap (yellow) except for native lymphatic vessels (arrowhead) or LYVE-1 monocytes (arrows) in the tumor septum of a proliferating IH from the ear of a 3-month-old female infant. (b) Histology of a serial section shows a lobular mass composed of capillaries. (c) Double stains for LYVE-1 and CD31 clearly reveals exclusive staining of dermal lymphatics (arrowhead) and tumor blood vessels, respectively, with virtually no overlap in a pyogenic granuloma from the thumb of a 4-year-old girl. (d) Histology of a serial section shows lobular proliferation of small capillaries, surrounded by fibromyxoid stroma. (e) Double stains for LYVE-1 and CD31 reveal intramuscular CD31 ${ }^{+}$, but LYVE-1 $1^{-}$tumor cells in an intramuscular hemangioma on the tongue of a 11-year-old female, whereas native lymphatics are LYVE-1 ${ }^{+}$(arrowheads). (f) Histology of a serial section shows tumor capillaries infiltrating in between skeletal muscle. (g) Double stains for LYVE-1 and CD31 reveal CD31+ , but LYVE-1 ${ }^{-}$tumor lobules, numerous stromal lymphatics are LYVE-1 $1^{+}$in a congenital nonprogressive hemangioma (CNH) from the forehead of a 3-month-old female (arrowheads). (h) Histology of a serial section reveals numerous lobules composed of proliferating capillaries. Cell nuclei are counterstained blue with Hoechst (a, c, e and g). Original magnifications: $\times 100(\mathbf{g})$ and $(\mathbf{h})$ and $\times 200(\mathbf{a})-(\mathbf{f})$.

Two pathologists (SSD and PEN) reconfirmed the diagnosis of all 62 vascular lesions.

\section{Immunofluorescent Stains}

Paraffin sections (6 $\mu \mathrm{m}$ thickness) were dewaxed, hydrated and treated with $0.01 \%$ protease XXIV (Sigma, St Louis, MO, USA) in phosphate-buffered saline for $20 \mathrm{~min}$ at $37^{\circ} \mathrm{C}$ for all 62 lesions. Sections were double stained using a rabbit polyclonal antibody against human LYVE- ${ }^{20}$ and a mouse monoclonal anti-human CD31 antibody (Table 2), followed by incubation with the respective secondary antibodies that were labeled with either Texas
Red (1:50) or with fluorescein isothiocyanate (1:50) (Jackson ImmunoResearch, West Grove, PA, USA), as previously described. ${ }^{22}$ Cell nuclei were counterstained with $20 \mu \mathrm{g} / \mathrm{ml}$ Hoechst bisbenzimide (Sigma). Additional immunohistochemical stains were performed using antibodies against CD34, Prox-1, GLUT-1, CD68 or CD11b (Table 2). Fresh-frozen tissue sections were incubated in acetone at $-20^{\circ} \mathrm{C}$ for $2 \mathrm{~min}$ and in $80 \%$ methanol at $4^{\circ} \mathrm{C}$ for $5 \mathrm{~min}$, blocked in $12 \%$ BSA solution for 20 min prior to incubation with primary antibody in four proliferating infantile hemangiomas, where paraffin section immunostaining for Prox1 and CD11b was not possible. Three observers scored the stained sections for semiquantitative LYVE-1 expression in 

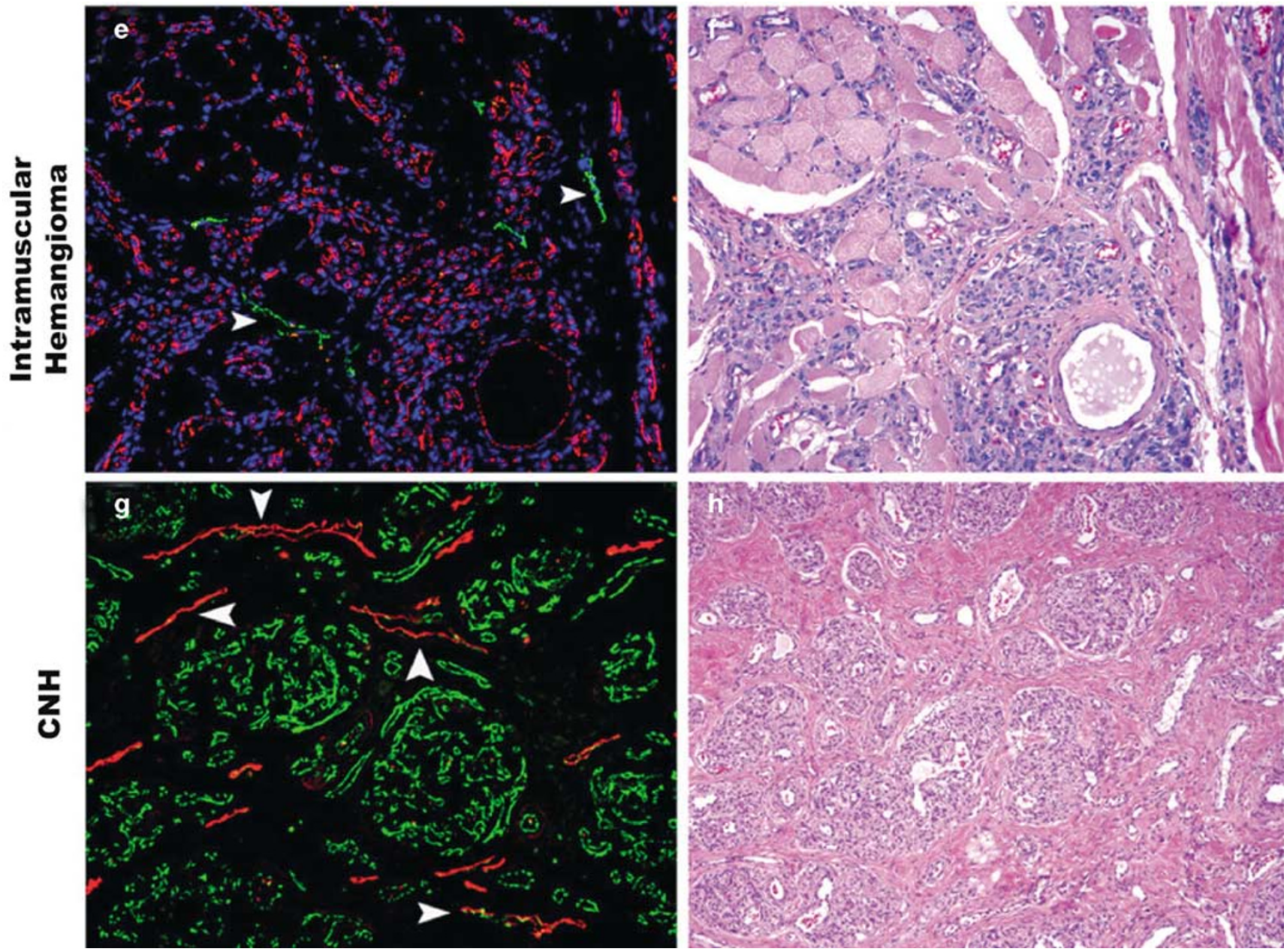

Figure 2 Continued.

Table 1 Patient characteristics with vascular lesions

\begin{tabular}{|c|c|c|c|c|c|c|}
\hline Diagnosis & Total & Age range & Mean age & Median age & Sex ratio F:M & Site $^{\text {a }} H N / T / U E / L E \%$ \\
\hline \multicolumn{7}{|l|}{ Infantile hemangioma } \\
\hline Proliferative phase & 19 & 3-18 month & 7.8 month & 8 month & $8.5: 1$ & $83.3 / 0 / 16.7 / 0$ \\
\hline Involutive phase & 9 & 2-60 months & 26.3 months & 22 months & $3.5: 1$ & $100 / 0 / 0 / 0$ \\
\hline Total & 28 & & & & & \\
\hline Pyogenic granuloma & 19 & $0.5-36$ years & 9.4 years & 8 years & $1.4: 1$ & $66.7 / 26.7 / 6.6 / 0$ \\
\hline Intramuscular hemangioma & 9 & $0.75-29$ years & 13.6 years & 11 years & $0.8: 1$ & $37.5 / 12.5 / 37.5 / 12.5$ \\
\hline Umbilical vascular malformation & 2 & - & - & - & - & $0 / 100 / 0 / 0$ \\
\hline Congenital nonprogressive hemangioma & 1 & 3 months & & & $1: 0$ & $100 / 0 / 0 / 0$ \\
\hline Reactive angioendotheliomatosis & 1 & 12 years & & & $0: 1$ & $0 / 0 / 100 / 0$ \\
\hline Synovial vascular malformation & 1 & 6 years & & & $0: 1$ & $0 / 0 / 0 / 100$ \\
\hline Cherry angioma & 1 & 45 years & & & $1: 0$ & $0 / 0 / 100 / 0$ \\
\hline Total & 62 & & & & & \\
\hline
\end{tabular}

${ }^{\mathrm{a}} \mathrm{HN} / \mathrm{T} / \mathrm{UE} / \mathrm{LE} \%$ indicates the respective percentages of specimens located on the head and neck, trunk, upper extremities, and lower extremities, respectively.

tumor cells, together with hematoxylin-eosinstained serial sections. The results were scored as follows, $1+$ : $<10 \%$ of lesional vessels positive; $2+$ : $10-50 \%$ of lesional vessels positive; $3+$ : $>50 \%$ of lesional vessels positive (Table 3 ).

\section{Statistical Analysis}

The unpaired Student's $t$-test was used to determine the statistical significance ( $P$-value) of LYVE-1 expression. 

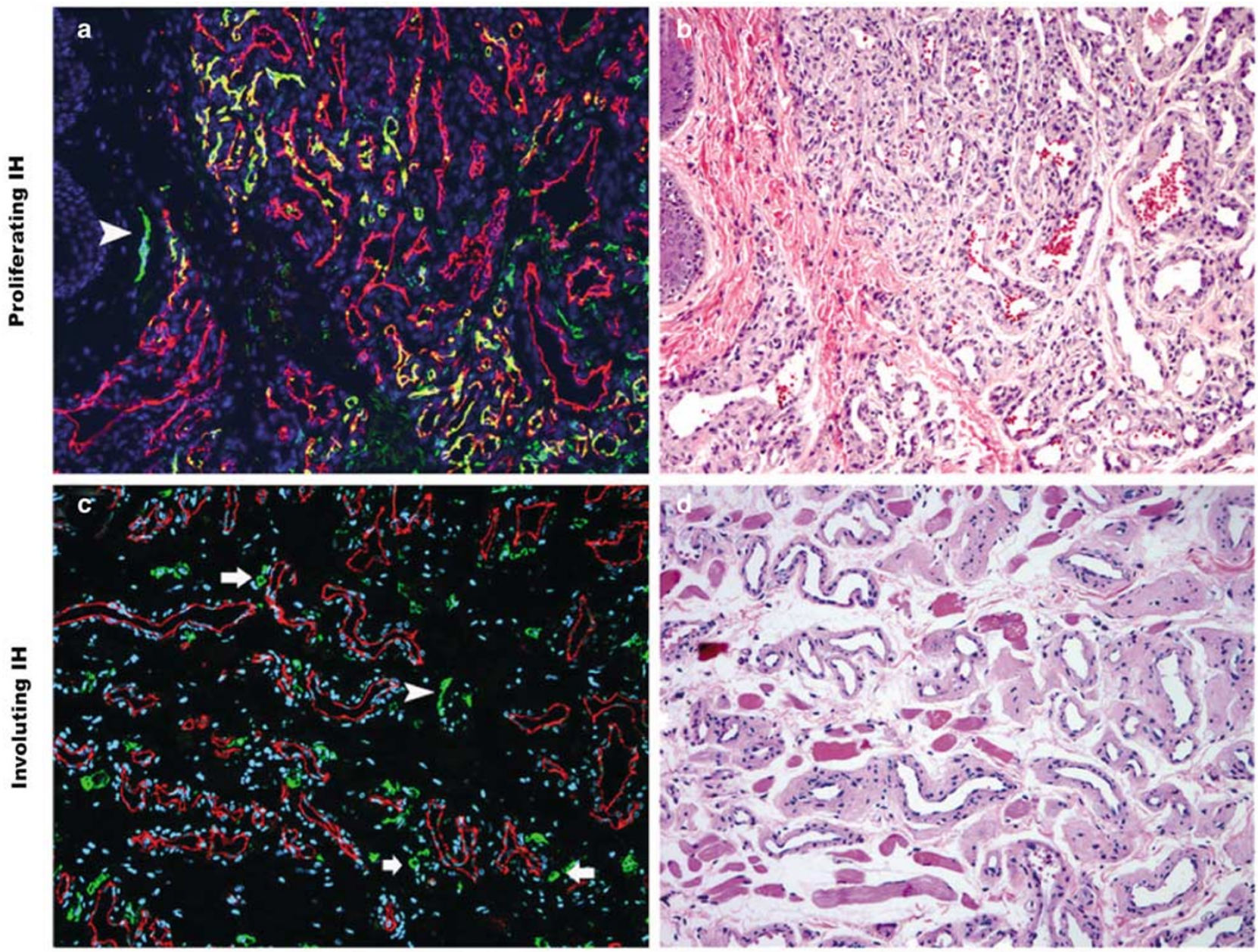

Figure 3 LYVE-1 expression is downregulated in infantile hemangiomas during involution. (a) and (e) Double immunofluorescent stain for LYVE-1 (green) and CD31 (red) shows overlap (yellow) in many endothelial cells of two proliferating infantile hemangiomas (IH) from the arm of an 8-month-old female infant and from the scalp of an 8-month-old female infant, respectively, except for native lymphatic vessels (arrowheads). (b) and (f) Histology of serial sections show lobules of vascular proliferation. (c) and (g) Double stains for LYVE-1 and CD31 reveal loss of LYVE-1 expression from all the tumor vessels in two involuting IH from the upper lip of a 1-year-old female child and from the scalp of an 8-month-old female infant, respectively, native lymphatics (arrowheads) and many monocytes (arrows) are LYVE-1 $1^{+}$(d) and (h) Histology of a serial section reveals absence of vascular proliferation and fibrofatty residuum. Cell nuclei are counterstained blue with Hoechst (a), (c), (e) and (g). Original magnifications: $\times 200(\mathbf{a})-(\mathbf{h})$.

\section{Results}

\section{LYVE-1 Expression by Endothelial Cells of Proliferating Infantile Hemangiomas}

To investigate the lineage-specific differentiation of infantile hemangioma, we examined tissue sections from patients with both proliferating and involuting infantile hemangiomas for markers of lymphatic and blood vascular endothelium. We found that the entire infantile hemangioma lobule showed endothelial positivity for the panendothelial marker CD31 (12) (Figure 1b). Surprisingly, the entire lobule was also strongly positive for LYVE-1 in most cases (Figure 1a). Merged images of LYVE-1 and CD31 revealed a virtually complete overlap of the green and red signals, suggesting that the lesional capillaries coexpressed both LYVE-1 and CD31 (Figure 1c). Normal blood vessels adjacent to infantile hemangiomas did not express LYVE-1 (Figure 1g).
We detected LYVE-1 expression in 19/19 (100\%) proliferating infantile hemangioma and 5/9 (55.6\%) involuting infantile hemangioma $(P=0.0009)$. Although all of the proliferating infantile hemangioma expressed LYVE-1, the lesional capillaries showed a variable immunoreactivity (Figure 1c, e and g). Based on the percentage of lesional capillaries, all tumors were assessed for semiquantitative LYVE-1 expression. The majority $(79 \%)$ of proliferating infantile hemangiomas showed either $>10-50 \%$ or $>50 \%$ of lesional vessels immunoreactive for LYVE-1 (Table 3). High magnification views of immunostains revealed both cytoplasmic and membranous distribution of LYVE-1 (Figure 1h).

To investigate whether LYVE-1 expression was specific for infantile hemangiomas, we examined several other benign vascular tumors for their LYVE-1 immunoreactivity. Compared to proliferating infantile hemangiomas, LYVE-1 expression was 

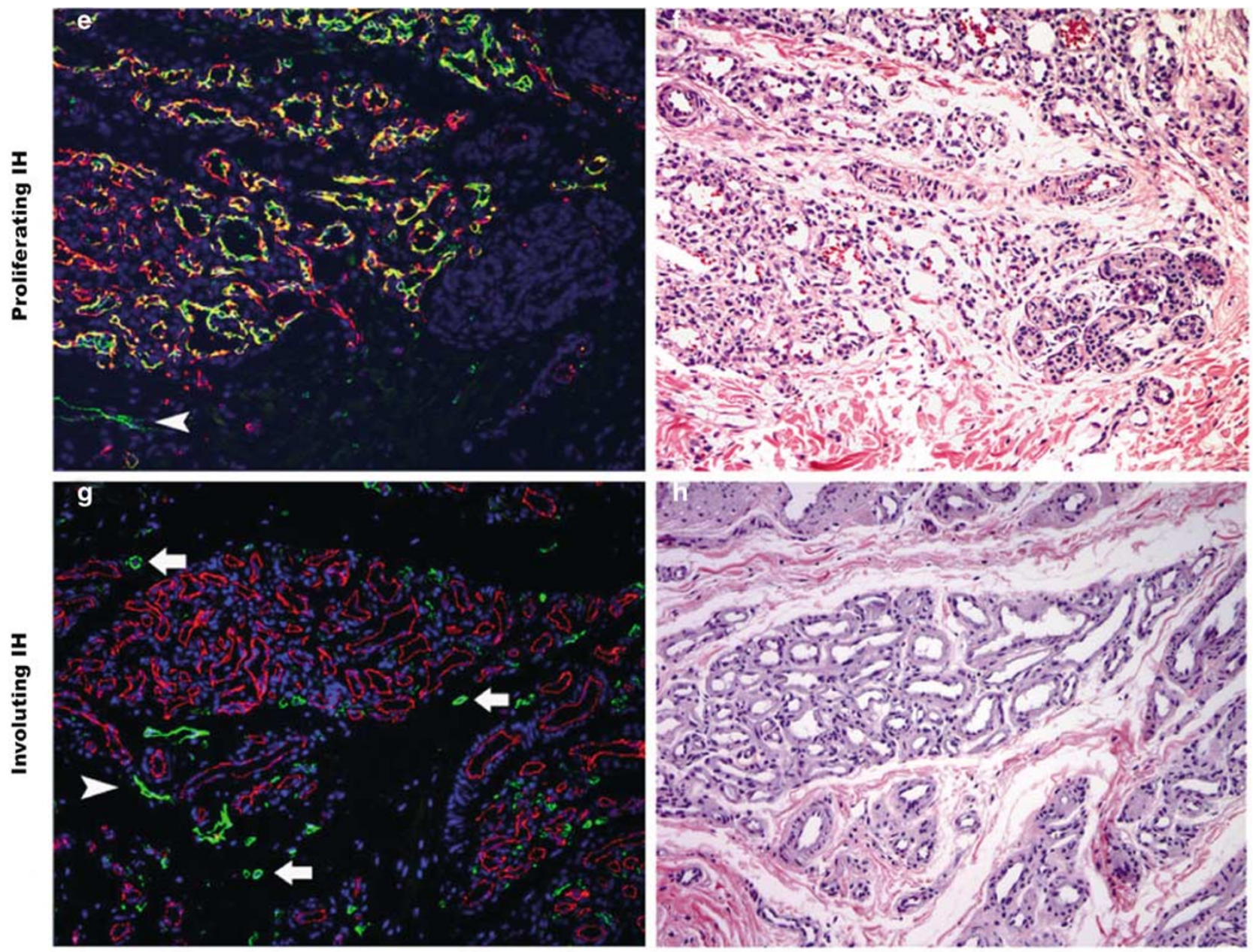

Figure 3 Continued.

Table 2 Antibodies used for immunofluorescent analysis

\begin{tabular}{|c|c|c|c|c|}
\hline Antibody & Source & Tуре & Tissue section & Dilution and conditions \\
\hline LYVE-1 & DG Jackson & Polyclonal & Paraffin and frozen & 1:600; O/N $\left(4^{\circ} \mathrm{C}\right)$ \\
\hline CD31 & DAKO & Monoclonal, IgG1 & Paraffin and frozen & 1:50; $3 \mathrm{~h}(\mathrm{RT})$ \\
\hline CD34 & DAKO & Monoclonal, IgG1 $\kappa$ & Paraffin & 1:100; $30 \min (\mathrm{RT})$ \\
\hline Glut-1 & DAKO & Polyclonal & Paraffin & 1:300; $30 \min (\mathrm{RT})$ \\
\hline CD68 & DAKO & Monoclonal, IgG1 & Paraffin & 1:50; $3 \mathrm{~h}$ (RT) \\
\hline CD11b & BD Pharmingen & Monoclonal, IgG1 $\kappa$ & Frozen & 1:50; $\mathrm{O} / \mathrm{N}\left(4^{\circ} \mathrm{C}\right)$ \\
\hline Prox-1 & K Alitalo & Polyclonal & Frozen & 1:100; $\mathrm{O} / \mathrm{N}\left(4^{\circ} \mathrm{C}\right)$ \\
\hline
\end{tabular}

RT, room temperature; O/N, overnight.

focally and weakly detected in only $3 / 19$ (15.8\%, $P<0.0001)$ PGs, in a pattern suggestive of normal, intra- and peri-lesional draining lymphatics, whereas the remainder of vascular lesions including intramuscular hemangiomas (9/9), umbilical vascular malformations (2/2), congenital nonprogressive hemangioma (1/1), synovial vascular malformation (1/1), cherry hemangioma (1/1) and reactive angioendotheliomatosis (1/1) were completely negative for LYVE-1. All examined vascular lesions were positive for CD31 (Figure 2). Interestingly, congenital nonprogressive hemangiomas are composed of numerous distinct proliferating capillaries admixed with many thin-walled vessels, suggestive of lymphatic vessels, ${ }^{24}$ which are in fact strongly LYVE-1 positive (Figure 2g). Umbilical and synovial vascular malformations were negative for GLUT-1 and LYVE-1 (data not shown). The age ranges for patients with other vascular tumors overlapped with those of the infantile hemangioma group ( 2 months to 

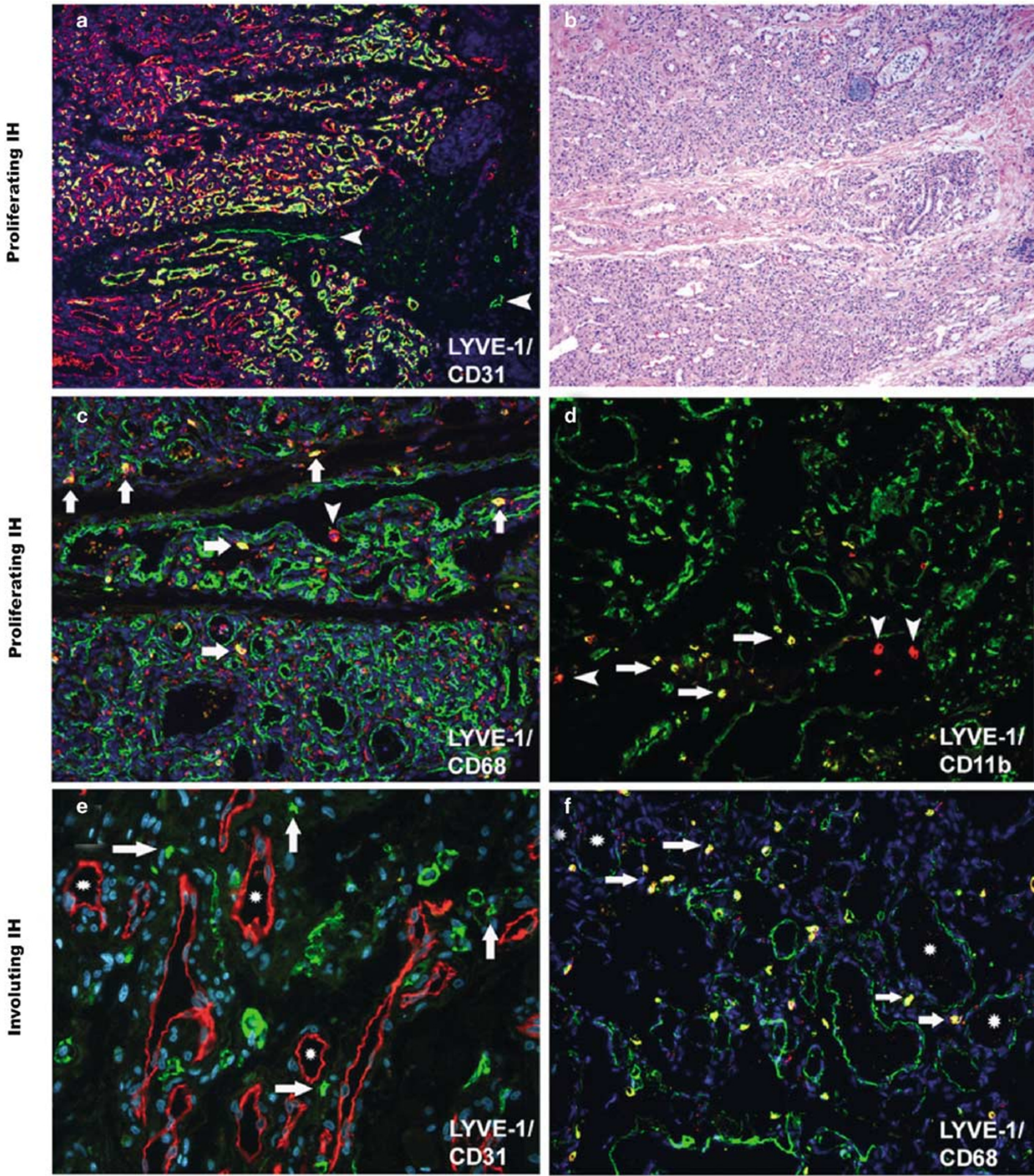

Figure 4 LYVE-1 ${ }^{+}$macrophages infiltrate infantile hemangiomas (IH). (a) Double immunofluorescent stain for LYVE-1 (green) and CD31 (red) shows extensive overlap (yellow) in many lesional capillaries of a proliferating IH from the ear of a 3-month-old female, except for native lymphatics (arrowheads). (b) Histology of a serial section shows marked lobular proliferation of capillaries. (c) Numerous LYVE$1^{+}$(green), CD68 (red) ${ }^{+}$macrophages (yellow) (arrows) are detected in a proliferating IH; lesional capillaries are LYVE-1 ${ }^{+}$. An intravascular LYVE-1- ${ }^{-}$CD68 $^{+}$granulocyte is seen (arrowhead). (d) Double stains for LYVE-1 (green) and CD11b (red) demonstrate numerous LYVE-1 ${ }^{+}$, CD11b ${ }^{+}$macrophages (yellow) (arrows) in a proliferating IH; lesional capillaries are LYVE-1 ${ }^{+}$. LYVE-1 ${ }^{-}$, CD11b ${ }^{+}$ granulocytes are seen (arrowheads). (e) Double stains for LYVE-1 and CD31 reveal complete loss of LYVE-1 expression from lesional vessels (asterisks) in an involuting IH. Numerous LYVE-1 $1^{+}$monocytes (arrows) are detected in the fibrofatty tissue. (f) Numerous LYVE$1^{+}, \mathrm{CD}_{11 \mathrm{~b}^{+}}$macrophages (yellow) (arrows) are detected, whereas lesional capillaries are LYVE-1 ${ }^{-}$(asterisks) in an involuting IH. Original magnifications: $\times 100(\mathbf{a})$ and $(\mathbf{b}), \times 200(\mathbf{c})$, (d) and (f), and $\times 400(\mathbf{e})$. 

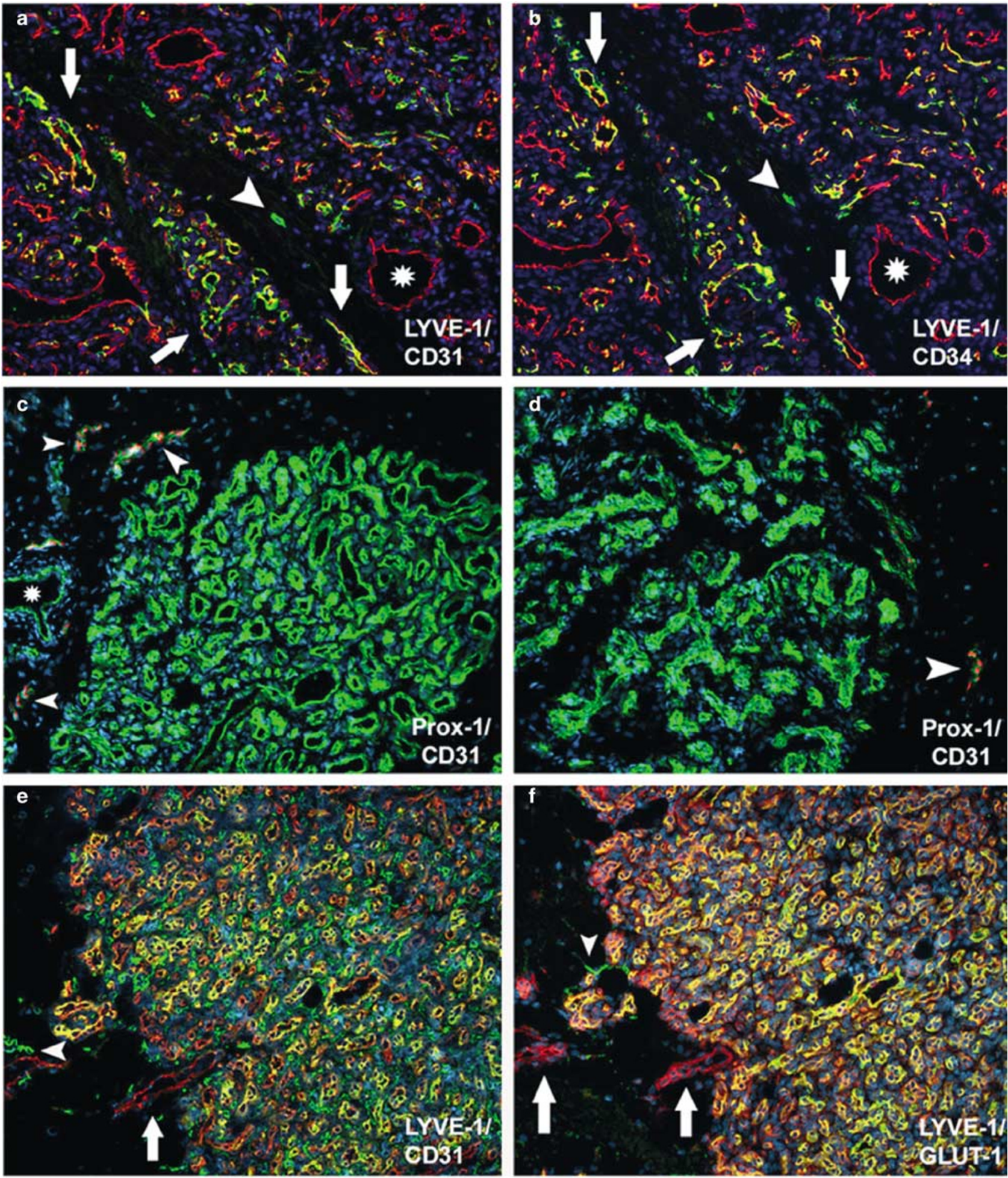

Figure 5 Proliferating infantile hemangiomas are LYVE-1+/CD34

(a) Double immunofluorescent stain for LYVE-1 (green) and CD31 (red) reveals that the great majority of lesional capillaries coexpress LYVE-1 and CD31 (yellow) (arrows) in proliferating infantile hemangiomas. (b) The very same LYVE-1+, CD31+ lesional capillaries are also positive for CD34 (yellow) (arrows) in a serial section. Native lymphatics are seen in the interlobular septum (arrowheads). Large CD31 ${ }^{+}$, CD34 $4^{+}$blood vessels do not express LYVE-1 (asterisk). (c) and (d) Double stains for Prox-1 (red) and CD31 (green) reveal that all tumor endothelial cells are completely negative for Prox-1 but strongly positive for CD31 in two proliferating infantile hemangiomas. Native lymphatics are seen at the tumor margin (arrowheads) and showed Prox $-1^{+}$nuclear and CD $31^{+}$cytoplasmic staining. In contrast, native CD31 ${ }^{+}$blood vascular (asterisk) endothelial cell nuclei are negative for Prox-1. (e) Double-staining for LYVE-1 (green) and CD31 (red) reveals LYVE-1 ${ }^{+} /$CD31 ${ }^{+}$lesional capillaries (yellow). (f) The very same LYVE-1 ${ }^{+} / \mathrm{CD} 31^{+}$lesional capillaries are also positive for GLUT-1 (yellow) in a serial section. Native lymphatics are seen at the tumor margin (arrowhead). Large blood vessels do not express LYVE-1 (arrows). Original magnifications: $\times 200$ (a)-(f). 
Table 3 Summary of LYVE-1 immunoreactivity in the lesional vessels

\begin{tabular}{|c|c|c|c|c|c|c|}
\hline Diagnosis & No. of specimens & Negative & Positive & $1+$ & $2+$ & $3+$ \\
\hline \multicolumn{7}{|l|}{ Infantile hemangioma } \\
\hline Proliferative phase & 19 & 0 & $19 *(100 \%)$ & $4(21 \%)$ & $9(47.4 \%)$ & $6(31.6 \%)$ \\
\hline Involutive phase & 9 & $4(44.4 \%)$ & $5 *(55.6 \%)$ & $3(33.4 \%)$ & $2(22.2 \%)$ & 0 \\
\hline Pyogenic granuloma & 19 & $16(84.2 \%)$ & $3 * *(15.8 \%)$ & $2(10.5 \%)$ & $1(5.3 \%)$ & \\
\hline Intramuscular hemangioma & 9 & $9(100 \%)$ & 0 & & & \\
\hline Umbilical vascular malformation & 2 & $2(100 \%)$ & 0 & & & \\
\hline Congenital nonprogressive hemangioma & 1 & $1(100 \%)$ & 0 & & & \\
\hline Reactive angioendotheliomatosis & 1 & $1(100 \%)$ & 0 & & & \\
\hline Synovial vascular malformation & 1 & $1(100 \%)$ & 0 & & & \\
\hline Cherry angioma & 1 & $1(100 \%)$ & 0 & & & \\
\hline Total & 62 & $35(56 \%)$ & $27(44 \%)$ & & & \\
\hline
\end{tabular}

${ }^{*} P$-value $=0.0009 ;{ }^{*} P$-value $<0.0001 .1+:<10 \%$ of lesional vessels positive; $2+: 10-50 \%$ of lesional vessels positive; $3+:>50 \%$ of lesional vessels positive. Percentage of positive cases is shown in parentheses.

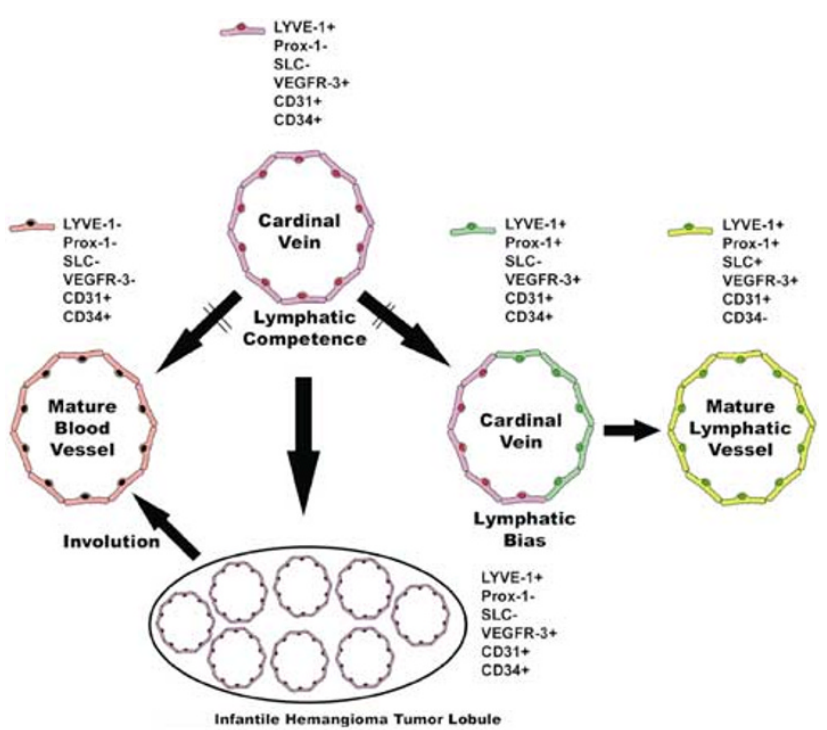

Figure 6 Arrested vascular development hypothesis. The lymphatic competence of the cardinal vein is defined by its endothelial cell immunophenotype: LYVE-1 ${ }^{+}$, Prox-1 ${ }^{-}$, SLC ${ }^{-}$ VEGFR- $3^{+}, \mathrm{CD} 1^{+}$and $\mathrm{CD} 34^{+}$, as are the infantile hemangioma cells. During normal embryogenesis, cardinal vein endothelial cells can mature into blood vessels or acquire a lymphatic bias, after onset of Prox-1 expression, to become mature lymphatic vessels. This maturity is defined by its endothelial cell immunophenotype: LYVE-1 $1^{+}$, Prox- $1+, \mathrm{SLC}^{+}, \mathrm{VEGFR}-3^{+}, \mathrm{CD} 31^{+}$and CD34- The endothelial cells in infantile hemangiomas display an abnormal immunophenotype, similar to that in cardinal vein with lymphatic competence, suggesting that these cells are arrested in an immature stage of vascular development, leading to their rapid proliferation. As LYVE-1 expression is downregulated, these tumors regress (involution) and develop into mature blood vessels.

5 years) (Table 1). Therefore, LYVE-1 expression is an age-independent phenomenon specific for endothelial cells of proliferating infantile hemangiomas.

\section{Downregulation of LYVE-1 Expression in Infantile Hemangiomas during Involution}

Analysis of infantile hemangiomas demonstrated absence of LYVE-1 immunoreactivity from the majority of tumor endothelial cells during involution, whereas CD31 expression remained unchanged (Figure 3). In fact, 4/9 (44.4\%) of involuting infantile hemangiomas were completely negative for LYVE-1 expression, and only a few lesional capillaries $(<10 \%)$ were LYVE-1 immunoreactive in the other cases in contrast to proliferating lesions $(P=0.0009)$ (Table 3). Involuting lesions displayed flattening of endothelium (Figure $3 \mathrm{~d}$ and $\mathrm{h}$ ), in contrast to the plump shape of endothelial cells during the proliferative phase (Figure $3 \mathrm{~b}$ and f). Although the lobular architecture was maintained in some involuting lesions (Figure 3h), LYVE-1 expression was lost as involution proceeded (Figure $3 \mathrm{~h}$ ).

\section{Detection of LYVE-1 Positive Macrophages in Infantile Hemangiomas}

We detected numerous LYVE-1 ${ }^{+}$mononuclear cells in both proliferating (Figure 2a) and involuting infantile hemangioma (Figure 3c and g). To further characterize these cells, we performed double stains with the macrophage markers, CD11 ${ }^{25}$ and CD68 ${ }^{26}$ and LYVE-1. We found that LYVE-1 ${ }^{+}$mononuclear cells expressed both CD11b and CD68 in proliferating and involuting infantile hemangiomas (Figure 4), identifying them as macrophages.

\section{Lesional Endothelial Cells are Arrested in Vascular Development}

Since lesional capillaries of infantile hemangiomas expressed LYVE-1, a specific lymphatic marker, we assessed these tumors for the expression of the lineage-specific lymphatic homeobox gene, Prox-1, and of the blood vascular-specific marker, CD34. Double immunofluorescence stains for LYVE-1/ CD31 and LYVE-1/CD34 in serial sections revealed that LYVE-1 ${ }^{+}$endothelial cells also express CD34 (Figure 5a and b). Double immunofluorescence stains of serial sections for LYVE-1/CD31 and Prox1/CD31 revealed that all tumor endothelial cells were negative for Prox-1 (Figure 5c and d). Hence, 
infantile hemangioma endothelial cells are LYVE-1 ${ }^{+}$, CD $31^{+}, \mathrm{CD}_{3} 4^{+}$but Prox-1- ${ }^{-}$. Double stains for LYVE1/ GLUT-1 clearly demonstrated that the tumor endothelial cells coexpressed both proteins during the proliferative phase (Figure 5e and f).

\section{Discussion}

Despite the high prevalence of infantile hemangiomas in the pediatric population, the molecular events leading to rapid proliferation and slow involution of these tumors remain unclear. We demonstrate here that the hyaluronan receptor LYVE-1 is specifically expressed in endothelial cells of infantile hemangioma during the proliferative phase and is downregulated during involution. Moreover, infantile hemangioma endothelial cells are Prox-1 ${ }^{-}, \mathrm{SLC}^{-}, \mathrm{VEGFR}^{-}{ }^{+}, \mathrm{CD} 31^{+}$and CD $34^{+}$. This endothelial cell immunophenotype resembles that of the cardinal vein during early normal embryogenesis, at a stage leading to the development of mature blood vessels or to acquiring lymphatic bias ${ }^{27}$ (Figure 6).

After the initial formation of the vascular system, a subpopulation of venous endothelial cells becomes competent to respond to a lymphatic inducing signal by expressing the homeobox gene Prox-1, at day $\sim$ E9.5. ${ }^{28}$ Our data suggest that the endothelial cells in infantile hemangiomas display an immature immunophenotype, similar to that in cardinal vein endothelial cell with lymphatic competence, suggesting the endothelial cells are arrested in an early stage of vascular development. This undifferentiated phenotype may explain their rapid proliferation. As infantile hemangiomas regress, the endothelial cells, coincident with downregulation of LYVE-1, attain a more mature immunophenotype, LYVE-1 ${ }^{-}$, Prox-1 ${ }^{-}$, SLC $^{-}$, VEGFR-3 ${ }^{-},{\text {CD } 31^{+}}^{+}$and $\mathrm{CD}_{34}{ }^{+}$, and develop into mature blood vessels (Figure 6). We hypothesize that somatic mutations may lead to arrested maturation and clonal expansion of infantile hemangioma endothelial cells with an immature immunophenotype.

The isolation of CD133+/CD34+ endothelial progenitor cells from proliferating infantile hemangiomas $^{8}$ supports the primitive nature of these endothelial cells, since CD133 is expressed by embryonic epithelium (neuroepithelium, kidney and gut $)^{29}$ and $\mathrm{CD} 4^{+}$endothelial cell precursors (hemangioblasts). ${ }^{30}$ It has been demonstrated that infantile hemangiomas share expression of GLUT-1, merosin, Fc $\gamma$ RII and Lewis $\mathrm{Y}$ antigen with human placenta microvasculature ${ }^{19}$ further supporting the fetal or embryonic nature of infantile hemangioma endothelial cells. Together, these results and our data suggest an intrinsic defect in infantile hemangioma endothelial cell maturation that may result in their abnormal proliferation.

In summary, our study demonstrates, for the first time, specific expression of LYVE-1 in infantile hemangioma endothelial cells during the proliferative phase and downregulation during the involuting phase. This identifies LYVE-1 as a novel marker for proliferating infantile hemangioma, since GLUT1 is expressed during both phases. ${ }^{19}$ Analysis of the specific blood and lymphatic endothelial cell markers, CD34 and Prox-1, respectively, revealed that the endothelial cells of infantile hemangioma have an immunophenotype similar in this regard to that of the embryonic vein during normal vascular development. We propose that infantile hemangioma endothelial cells are arrested in an early stage of vascular differentiation, with important implications for the pathogenesis of rapid postnatal proliferation.

\section{Acknowledgements}

We thank L Janes, L Nguyen and A Mizeracki for expert technical assistance; K Alitalo for providing Prox1 antibodies; and DG Jackson for the gift of LYVE-1 antibody. This work was supported by NIH/ NCI Grants CA69184, CA86410 and CA91861 (MD), by an NIH Pathology Training Grant (SSD), and by the Cutaneous Biology Research Center through the Massachusetts General Hospital/Shiseido Co. Ltd. Agreement (MD).

\section{References}

1 Garzon M. Infantile hemangiomas. In: Bolognia J, Jorizz J, Rapini RP (eds). Dermatology. Mosby: London, 2003, pp 1599-1614 Chapter 104.

2 Bruckner AL, Frieden IJ. Hemangiomas of infancy. J Am Acad Dermatol 2003;48:477-493.

3 Margileth AM, Museles M. Cutaneous hemangiomas in children. Diagnosis and conservative management. JAMA 1965;194:523-526.

4 North PE, Waner M, Mizeracki A, et al. GLUT1: a newly discovered immunohistochemical marker for juvenile hemangiomas. Hum Pathol 2000;31:11-22.

5 Mulliken JB, Glowacki J. Hemangiomas and vascular malformations in infants and children: a classification based on endothelial characteristics. Plast Reconstr Surg 1982;69:412-422.

6 Boye E, Yu Y, Paranya G, et al. Clonality and altered behavior of endothelial cells from hemangiomas. J Clin Invest 2001;107:745-752.

7 Walter JW, North PE, Waner M, et al. Somatic mutation of vascular endothelial growth factor receptors in juvenile hemangioma. Genes Chromosomes Cancer 2002;33:295-303.

$8 \mathrm{Yu} \mathrm{Y,} \mathrm{Flint} \mathrm{AF,} \mathrm{Mulliken} \mathrm{JB,} \mathrm{et} \mathrm{al.} \mathrm{Endothelial}$ progenitor cells in infantile hemangioma. Blood 2003, prepublished online, October 23.

9 Bischoff J. Monoclonal expansion of endothelial cells in hemangioma: an intrinsic defect with extrinsic consequences? Trends Cardiovasc Med 2002;12: $220-224$. 
10 Walter JW, Blei F, Anderson JL, et al. Genetic mapping of a novel familial form of infantile hemangioma. Am J Med Genet 1999;82:77-83.

$11 \mathrm{Yu}$ Y, Varughese J, Brown LF, et al. Increased Tie2 expression, enhanced response to angiopoietin-1, and dysregulated angiopoietin-2 expression in hemangioma-derived endothelial cells. Am J Pathol 2001; 159:2271-2280.

12 Takahashi K, Mulliken JB, Kozakewich HP, et al. Cellular markers that distinguish the phases of hemangioma during infancy and childhood. J Clin Invest 1994;93:2357-2364.

13 Chang J, Most D, Bresnick S, et al. Proliferative hemangiomas: analysis of cytokine gene expression and angiogenesis. Plast Reconstr Surg 1999;103:1-9.

14 Isik FF, Rand RP, Gruss JS, et al. Monocyte chemoattractant protein-1 mRNA expression in hemangiomas and vascular malformations. J Surg Res 1996;61:71-76.

15 Ritter MR, Dorrell MI, Edmonds J, et al. Insulin-like growth factor 2 and potential regulators of hemangioma growth and involution identified by large-scale expression analysis. Proc Natl Acad Sci USA 2002;99:7455-7460.

16 Ezekowitz RA, Mulliken JB, Folkman J. Interferon alfa2a therapy for life-threatening hemangiomas of infancy. N Engl J Med 1992;326:1456-1463.

17 Martinez MI, Sanchez-Carpintero I, North PE, et al. Infantile hemangioma: clinical resolution with 5\% imiquimod cream. Arch Dermatol 2002;138:881-884 discussion 884 .

18 Bielenberg DR, Bucana CD, Sanchez R, et al. Progressive growth of infantile cutaneous hemangiomas is directly correlated with hyperplasia and angiogenesis of adjacent epidermis and inversely correlated with expression of the endogenous angiogenesis inhibitor, IFN-beta. Int J Oncol 1999;14:401-408.

19 North PE, Waner M, Mizeracki A, et al. A unique microvascular phenotype shared by juvenile hemangiomas and human placenta. Arch Dermatol 2001;137:559-570.

20 Banerji S, Ni J, Wang SX, et al. LYVE-1, a new homologue of the CD44 glycoprotein, is a lymph- specific receptor for hyaluronan. J Cell Biol 1999;144:789-801.

21 Beasley NJ, Prevo R, Banerji S, et al. Intratumoral lymphangiogenesis and lymph node metastasis in head and neck cancer. Cancer Res 2002;62: 1315-1320.

22 Dadras SS, Paul T, Bertoncini J, et al. Tumor lymphangiogenesis: a novel prognostic indicator for cutaneous melanoma metastasis and survival. Am J Pathol 2003;162:1951-1960.

23 Jacobs A. Strawberry hemangiomas: the natural history of the untreated lesion. Calif Med 1957;86 8-10.

24 North PE, Waner M, James CA, et al. Congenital nonprogressive hemangioma: a distinct clinicopathologic entity unlike infantile hemangioma. Arch Dermatol 2001;137:1607-1620.

25 Corbi AL, Kishimoto TK, Miller LJ, et al. The human leukocyte adhesion glycoprotein Mac-1 (complement receptor type 3 , CD11b) alpha subunit. Cloning, primary structure, and relation to the integrins, von Willebrand factor and factor B. J Biol Chem 1988;263:12403-12411.

26 Holness CL, Simmons DL. Molecular cloning of CD68, a human macrophage marker related to lysosomal glycoproteins. Blood 1993;81:1607-1613.

27 Oliver G, Detmar M. The rediscovery of the lymphatic system: old and new insights into the development and biological function of the lymphatic vasculature. Genes Dev 2002;16:773-783.

28 Wigle JT, Oliver G. Prox1 function is required for the development of the murine lymphatic system. Cell 1999;98:769-778.

29 Corbeil D, Roper K, Hellwig A, et al. The human AC133 hematopoietic stem cell antigen is also expressed in epithelial cells and targeted to plasma membrane protrusions. J Biol Chem 2000;275 5512-5520.

30 Peichev M, Naiyer AJ, Pereira D, et al. Expression of VEGFR-2 and AC133 by circulating human CD34 (+) cells identifies a population of functional endothelial precursors. Blood 2000;95:952-958. 